\title{
Islamisme et violence : le cas de la Palestine (Partie
}

3)

Séverine Labat

\section{(2) OpenEdition \\ 1 Journals}

Édition électronique

URL : http://journals.openedition.org/conflits/702

DOI : $10.4000 /$ conflits.702

ISSN : $1777-5345$

Éditeur :

CCLS - Centre d'études sur les conflits lilberté et sécurité, L'Harmattan

Édition imprimée

Date de publication : 15 octobre 1998

ISSN : 1157-996X

Référence électronique

Séverine Labat, «Islamisme et violence : le cas de la Palestine (Partie 3) », Cultures \& Conflits [En ligne], 29-30 | automne-hiver 1998, mis en ligne le, consulté le 30 mars 2021. URL : http://

journals.openedition.org/conflits/702 ; DOI : https://doi.org/10.4000/conflits.702

Ce document a été généré automatiquement le 30 mars 2021.

Creative Commons License 


\title{
Islamisme et violence : le cas de la Palestine (Partie 3)
}

\author{
Séverine Labat
}

\section{La subjectivation par l'islam}

Du temps de l'Intifada, l'islam politique est parvenu à doter de larges pans du corps social d'une image revalorisée d'eux-mêmes en même temps qu'il leur permettait d'acquérir la conviction d'une utilité sociale.

L'incapacité du Hamas à peser par la suite sur les processus politiques, sociaux et culturels en cours représente l'un des motifs du passage à l'acte d'acteurs s'estimant désormais dépossédés de toute possibilité d'action dans les champs sociaux et politiques (à l'échelle nationale comme à l'échelle transnationale), alors même que les effets de l'occupation et de la répression demeurent toujours aussi prégnants.

\section{Libération et Islamisation}

En proposant des prolongements culturels à leur identité sociale, l'idéologie islamiste permet à ses partisans d'accéder à une forme de subjectivation par réappropriation de la définition de soi.

Aspirant à transcender les frontières nationales et transnationales de l'exclusion, les acteurs islamistes développent en effet une représentation du monde offrant la possibilité de sublimer la frustration de n'avoir pas accès aux fruits de la modernité.

A leurs yeux, l'Occident n'est parvenu à assurer sa domination politique, économique et culturelle, qu'à travers l'imposition d'un modèle de consommation aux effets dissolvants et corrupteurs en termes de cohésion sociale.

Fustigeant l'illusion consumériste des promoteurs d'un accord de paix qui, avant que ne soit retombé l'enthousiasme des premiers temps, vantaient les mérites économiques d'un futur " grand marché du Proche-Orient ", en contradiction flagrante avec les actuelles réalités socio-économiques, les acteurs islamistes peuvent opposer leur repli identitaire et leur exigence morale à la mondialisation et à la globalisation.

Proposant de libérer du joug israélien à la fois la terre de Palestine et les âmes qui la peuplent, l'idéologie islamiste fonctionne comme une sorte de " théologie de la 
libération "1 au sein de laquelle le " réarmement moral " joue un rôle décisif dans l'affranchissement de toute aliénation et dans la résistance à la dépossession de soi induite par l'occupation.

Durant les années d'Intifada, le Hamas s'est efforcé de canaliser la rage sociale d'une partie de la jeunesse palestinienne, tout en se faisant l'expression de sa volonté de participation au changement social et politique. Sa naissance avait tempéré les violences exercées auparavant par des militants islamistes à l'encontre d'autres militants politiques palestiniens (notamment communistes) et, de concert avec les autres forces engagées dans la lutte de libération, il contribuait à imposer un sévère contrôle social sur une population entièrement mobilisée autour de la cause nationale.

Durant le soulèvement, les forces politiques palestiniennes dans leur ensemble intervenaient dans la célébration des mariages, dans le prononcé des divorces, dans le règlement des conflits $\mathrm{d}$ 'héritage, mais se faisaient également les championnes de la lutte contre les " déviances " qu'étaient, à leurs yeux, la prostitution, l'homosexualité, la consommation d'alcool et de drogue (autant de " faiblesses " censées favoriser les " retournements " par les services israéliens). A l'instar des branches armées des autres formations palestiniennes ${ }^{2}$, les Brigades Azzeddine El-Qassem du Hamas s'étaient spécialisées dans l'assassinat de " collaborateurs " accusés " d'intelligence avec l'ennemi " ou, plus simplement, suspectés " d'empoisonner l'âme de la nation en profitant de leur fonction de journalistes, d'écrivains, d'éducateurs ou de n'importe quel autre moyen ".

" Pendant l'Intifada, nous étions des orphelins. Personne ne contrôlait nos affaires civiles. Nous avions à prendre en charge nos lois. Et les dealers étaient souvent liés à Israël. Ils collaboraient. Il fallait détruire le phénomène. Il fallait détruire la prostitution qui était une création de l'occupation. Il fallait détruire la corruption de notre société. Nous avons utilisé la violence. Nous avons tué des collaborateurs. Tout le monde l'a fait. Même le Fatah. Car l'occupation essayait de détruire notre communauté par tous les moyens "3.

Plus disciplinés que ceux du Fatah et du Front Populaire, les militants du Hamas ont rarement opéré hors du cadre fixé par leur organisation. En revanche, durant les années 1991-1992, des gangs formés de petits " seigneurs de la guerre " issus des rangs du Fatah (" Faucons " et " Panthères Noires " à Naplouse) et du FPLP (" Aigles Rouges ") ont fait la loi à Jénine et à Naplouse et développé des formes de résistance sociale se diluant de plus en plus dans des activités délictueuses. S'estimant " trahis " par la conférence de Madrid, privés d'encadrement depuis que, considérablement affaiblie, l'OLP investissait davantage la scène diplomatique que le champ social et politique intérieur, ils rackettaient la population par la levée d'un " impôt révolutionnaire " et procédaient à des liquidations sommaires de leurs adversaires, sous couvert de lutte contre les " collaborateurs ". Il fallut attendre le retour de Yasser Arafat pour que soit mis un terme à leurs activités et que la majorité d'entre eux intègrent les services de sécurité.

Quoiqu'il en soit, leur participation à l'Intifada, dans les rangs du Hamas comme dans ceux des autres organisations, a structuré la vie sociale de toute une génération de jeunes Palestiniens. Elle leur a permis de recouvrer une identité et une dignité mises à mal par trente ans d'occupation et de répression coloniales.

La participation des jeunes islamistes à la résistance armée se situe à la croisée entre violence sociale et violence politique, sans exclure des dérives de type criminel ou 
infra-politique, et au carrefour entre violence instrumentale (qui permet de prouver sa capacité de nuire lorsque l'on est privé d'autres ressources politiques) et violence expressive (mode d'affirmation de soi et d'affirmation de son aptitude à faire front). Elle met également en oeuvre des "violences verticales " ou " révolutionnaires " (tournées contre l'occupant mais également contre des concurrents politiques) et des " violences horizontales " ou " privées " (dirigées contre ceux qui sont accusés de contrevenir à la norme religieuse "4), tout en demeurant articulée à un projet politique de libération des territoires occupés.

L'abandon de la lutte armée : ou les illusions perdues de l'Intifada

La signature des accords d'Oslo et l'abandon des mots d'ordre de la lutte armée, alors même que les conditions de l'occupation demeurent, vont peu à peu accréditer, auprès d'une large fraction de la jeunesse défavorisée, l'idée suivant laquelle l'autonomie représente un nouveau mode d'exercice de l'occupation fondé sur la délégation et le contrôle à partir de l'extérieur ${ }^{5}$. Perçue par ses opposants comme une simple courroie de transmission, la nouvelle Autorité Palestinienne doit à la fois convaincre les dirigeants israéliens et occidentaux de son aptitude à maîtriser les territoires qu'elle administre (ce qui implique qu'elle se soumette à leurs injonctions en matière de répression contre la mouvance islamiste), et ménager une population palestinienne, certes durement éprouvée par sept ans de guerre, mais désormais en partie mobilisée par des organisations politiques rivales du Fatah.

Frustrée d'une victoire dont elle estime avoir été dépossédée par ceux de " l'extérieur " au détriment des " vrais combattants " du soulèvement, frustrée du sentiment d'exister dont l'armait sa participation à la lutte (la construction de l'Etat national mobilisant évidemment d'autres réseaux que ceux qui combattaient dans la " révolution des pierres "), sans pour autant être associée à un projet de participation politique, ni voir son activisme réinvesti dans la promotion d'un véritable projet national, une partie de la " génération de l'Intifada " conçoit, à l'égard du processus de paix, une amertume expliquant bien des passages à l'acte.

Il n'était que de voir, durant la crise de septembre 1996, la promptitude avec laquelle, sur le fond de cette désillusion vis à vis de la pax americana ${ }^{6}$, les jeunes participèrent à des affrontements qui leur rappelaient " le bon vieux temps de l'Intifada ".

Une partie des " enfants de l'Intifada ", qui avaient une dizaine d'années en 1987, et ont passé leur enfance et leur adolescence à participer aux temps forts du soulèvement populaire, se retrouvent désormais démobilisés, sans bagage scolaire ni emploi. Se pose, avec une acuité toute particulière, le problème de leur réinsertion dans la vie civile. Ceux qui n'ont pas intégré l'appareil policier palestinien, et qui ont pour seul capital social et symbolique leurs anciens faits d'armes (bien qu'exciper de sa participation à l'Intifada ait tendance à ne plus être vraiment valorisant), se sentent exclus d'un processus dont ils n'ont reçu aucun dividende.

" Les gens ont souffert pendant huit ans d'Intifada. mais cela s'est évaporé. Après huit ans de sacrifices, ils attendaient beaucoup. Le résultat est très faible et limité. L'Intifada a restauré l'honneur des Palestiniens, leur a redonné confiance en leur capacité à résister. L'Intifada a joué un rôle-clé. Si on n'avait pas été là, la situation du MoyenOrient serait différente. Ce qui se passe aujourd'hui est le résultat de l'action de notre génération. Surtout celle qui était au Hamas. C'est la menace que le Hamas a fait peser sur la sécurité d'Israël qui a conduit à l'accord. Mais cet accord s'est fait contre cette 
génération. C'était pour vaincre la génération qui s'était battue. Après la fierté, c'est la désillusion "7.

Colonisation et répression ont, durant les sept ans du soulèvement, unis les Palestiniens, toutes classes et toutes générations confondues. Aussi bien une partie de la jeunesse socialisée durant l'Intifada éprouve-t-elle les pires difficultés à surmonter les contradictions existant entre l'idéal égalitaire d'une société indifférenciée véhiculé par les discours populistes de l'Intifada (qu'il soit islamistes ou nationalistes), et le retour des contradictions de classes et des luttes socio-politiques - entre ceux qui ont intégré les rouages de l'Autorité Palestinienne et ceux qui ne s'y trouvent pas - entre l'idéal consensuel de ces années de feu et les réalités de la pratique du pouvoir ${ }^{8}$. C'est dans ce contexte que les conflits sociaux inter-palestiniens mais aussi transnationaux vont se reformuler dans le répertoire culturel. Privés de toute perspective en dehors de l'illusion de résistance qu'entretiennent les islamistes radicaux, ils idéalisent la période d'une Intifada marquée du sceau de la " moralité ", par contraste avec l'amoralité supposée des nouveaux dirigeants palestiniens.

" L'Intifada a été une aventure. Un risque qu'on a pris. Mais un groupe a utilisé l'Intifada pour en tirer des résultats. Cet accord a eu lieu lorsque l'intifada a commencé à se transformer en lutte armée. Ceux qui en ont récolté les fruits venaient de l'extérieur. Cet accord a été une trahison de l'intifada car il l'a empêché de réaliser tous ses objectifs. Ceux qui se sont sacrifiés ont perdu (...) J'aurais aimé ne pas avoir lancé de pierres étant donné le résultat. Je vivais avec dignité pendant l'occupation et maintenant, je ne vis plus dans la dignité. J'ai porté les rêves du peuple palestinien. Le Fatah disait vouloir la même chose, mais il y a des différences. Je veux ce qu'il y a de meilleur pour mon pays et je veux donc lui éviter le spectre de la guerre civile. Mais le Fatah fait des déclarations contradictoires depuis Madrid. Il insulte les gens du Hamas, les attaque à l'intérieur des prisons. Ils ont changé à $180^{\circ}$. De combattants, ils sont devenus les serviteurs d'Israël. Maintenant que l'Autorité est là, ils arrêtent les gens, ils les torturent de façon plus atroce que ne le faisaient les Israéliens. Tout cela contre ceux qui se sont sacrifiés et ont sacrifié leur argent pour leur pays (...). Le Hamas voulait continuer le djihad contre les Israéliens et en faire profiter à l'OLP en tant que carte de son jeu. Mais le Hamas a été déçu lorsque l'OLP a commencé à s'opposer publiquement au Hamas. Plutôt que de coopérer avec le Hamas contre les Israéliens, ils ont coopéré avec les Israéliens contre le Hamas. Et le dialogue entre l'Autorité et le Hamas est un dialogue de sourds. Cela n'a aucun intérêt pratique pour le peuple ou pour le mouvement. Les islamistes sont réprimés. Des gens sont en prison sans raison (...) L'Autorité est le principal obstacle à la poursuite de la lutte armée contre Israël (...) Il faut attendre que l'Autorité se plante et coule. Il faut se préparer à une autre lutte armée quand les circonstances s'y prêteront " 9 .

Aussi bien l'ambiance de relative détente - encore réservée à une élite privilégiée - qui règne actuellement en Cisjordanie mais également dans les quartiers les plus riches de Gaza, région traditionnellement la plus conservatrice de Palestine ${ }^{10}$, provoque-t-elle une certaine rancoeur à l'égard de ceux qu'une partie des Palestiniens nomment désormais les " étrangers ", les " Tunisiens " ou " ceux de Beyrouth ". Et, les responsables extérieurs sont perçus, par une partie de la jeunesse des camps de réfugiés, comme une greffe étrangère véhiculant des idées et des moeurs allogènes, et favorisant, par leur adoption du modèle de consommation occidental, la pénétration des modes de domination étrangers et de leur " relais " israélien. 
" Nous n'utilisons pas la force pour obliger les gens à être religieux. Chacun est libre d'être laïc ou religieux. Mais, si nous pouvions, nous lutterions contre les déviances que sont la drogue ou la prostitution. Mais l'Autorité ne le permet pas. L'Autorité veut changer la société. Ceux qui viennent de l'extérieur sont familiers d'autres valeurs. Les femmes portent des pantalons, ils boivent de l'alcool. Ce n'est pas familier des traditions de Gaza. Ils ont vécu dans d'autres circonstances. Surtout à Beyrouth, où il y avait plus de permissivité "11.

A cela s'ajoute le contraste entre la proclamation de la fin de la lutte armée et la réalité de la poursuite de l'occupation.

Les accords d'autonomie qui, en septembre 1993, avaient concédé aux Palestiniens l'administration " limitée " de sept " confettis " représentant environ six pour cent des territoires occupés, sont constamment violés par l'occupant israélien, alors qu'en 1996, auraient dû débuter les négociations sur le statut final des territoires autonomes ${ }^{12}$.

Quelque quatre à cinq mille Palestiniens sont toujours détenus dans les geôles israéliennes bien que leur élargissement ait été prévu par les accords.

Le plan de redéploiement de la ville arabe d'Hébron (cent vingt mille habitants palestiniens pris en otages par quatre cent colons juifs extrémistes dont la présence, au coeur de la ville, requiert la mobilisation de près de mille cinq cents soldats israéliens), négocié avec l'ancien gouvernement travailliste et prévu pour le mois de mars $1996^{13}$, n'a finalement fait l'objet d'un accord que le 15 janvier 1997. Celui-ci ne prévoit le retrait de l'armée israélienne que des quatre cinquièmes du territoire de la ville. Concernant les étapes futures du redéploiement (qui doit s'échelonner d'ici la mi-1998), l'accord signé par Yasser Arafat et Benjamin Netanyahou n'en précise pas l'amplitude, l'estimation de la superficie des territoires devant être évacués par l'armée israélienne ayant été laissée à la seule appréciation de l'Etat juif ${ }^{14}$. Et il n'est que de mentionner les divergences d'évaluation quant à l'ampleur que devrait revêtir ce retrait - 85 à $90 \%$ de la Cisjordanie selon les Palestiniens, 40 à $50 \%$ selon les Israéliens - pour mesurer l'étendue du malentendu...

Depuis le bouclage imposé depuis 1989, renforcé en mars 1993, puis rendu " total " à la suite des attentats-suicides de février-mars 1996, un régime d'apartheid sévit à l'encontre des populations palestiniennes soumises à un état de siège permanent ${ }^{15}$, tandis que la politique de colonisation juive des territoires occupés connait un net regain (politique d'expropriation des terres) ${ }^{16}$. Les " passages protégés " qui, conformément aux accords d'Oslo 2, doivent relier Gaza à la Cisjordanie (distantes de quatre-vingt-dix kilomètres), n'ont toujours pas fait l'objet d'un accord, ce qui réduit les territoires autonomes palestiniens à une série de " bantoustans " désarticulée et étroitement contrôlée par Israël, alors même que les colonies israéliennes sont désormais reliées entre elles par un système intégré de " routes de contournement " réservées à l'usage exclusif des colons ${ }^{17}$.

Plus, la mise en œuvre d'une politique visant à réduire le nombre de résidents non juifs de Jérusalem (la partie arabe de la ville, Jérusalem-Est, fut conquise en 1967 et annexée en 1980) chasse nombre de Palestiniens de ce que les Israéliens déclarent être la " capitale éternelle du peuple juif "18. Cette judaïsation ${ }^{19}$ de la ville passe notamment par la suppression des cartes d'identité au prétexte que le véritable " lieu de vie " de leurs détenteurs serait ailleurs, par le refus d'enregistrer les nouveaux-nés palestiniens nés en dehors du pays ou en dehors des frontières de Jérusalem sur la carte d'identité de leurs parents, par le refus de délivrer des cartes d'identité aux résidents de Jérusalem 
effectuant leurs études à l'étranger, et par la destruction des maisons arabes (quatrevingt-treize maisons ont été détruites sous l'administration travailliste) au motif que leurs propriétaires ne détiendraient pas de permis de construire en bonne et due forme (lesquels permis de construire ne sont guère délivrés aux Arabes). Il en résulte une situation d'asphyxie progressive : les entreprises palestiniennes ferment les unes après les autres (le bouclage interdisant aux marchandises et aux employés de passer) et sont contraintes de s'installer à Ramallah, ce qui a pour effet de priver leurs responsables de leur droit à la carte de résident de Jérusalem.

La répression menée par les services de sécurité israéliens joue également un rôle non négligeable dans le passage à l'acte de certains militants islamistes radicalisés. En effet, tandis que Hamas et le Djihad Islamique avaient suspendu leurs attaques armées depuis l'attentat du 21 août 1995, l'assassinat le 26 octobre à Malte de Fathi Chiqaqi (chef de la principale branche du Djihad Islamique), puis celui de Yahia Ayache le 5 janvier suivant, sont venus relancer la vague d'attentats à l'intérieur de l'ancienne ligne verte $^{20}$. Par ailleurs, les punitions collectives infligées par les autorités israéliennes à la suite de chaque nouvelle opération militaire (bouclage des territoires, destruction des maisons des familles de ceux qui sont impliqués dans des actes de violence) entretiennent à leur tour la spirale de la violence.

Résistance armée ou dérive terroriste?

Aussi bien, du fait de la légitimité qu'a acquise la lutte armée au cours de l'histoire du mouvement national, les opérations militaires du Hamas reçoivent-elles - du moins jusqu'au printemps 1996, puis à nouveau en mars 1997, après que le gouvernement israélien eut entrepris, (en violation des accords de paix) l'implantation d'une colonie juive à Abou Ghneim (Har Homa) dans la partie arabe de Jérusalem - l'approbation tacite et silencieuse d'une grande partie du corps social palestinien qui y discerne, le plus souvent, des actes de résistance politique.

Le primat de la cause nationale interdit en effet aux autres organisations politiques de condamner les opérations militaires anti-israéliennes du Hamas, considéré comme partie prenante de la lutte nationale, même si les mêmes forces politiques condamnent les velléités de certains dirigeants du Hamas de vouloir imposer leur ordre moral par la force.

Les groupes armés islamistes continuant d'incarner les aspirations d'une partie de la communauté palestinienne, et les opérations militaires du Hamas jouissant, aux yeux d'une partie de la société palestinienne, d'une certaine légitimité, les attentats antiisraéliens ne peuvent être réduits à de pures dérives terroristes.

Il est, à cet égard, symptomatique que les attentats islamistes aient cessé dès lors que la population palestinienne a commencé à en condamner les effets. Les attentats de février 1996, consécutifs à l'assassinat de Yahia Ayache par le Mossad, furent considérés comme une vengeance " légitime " à l'encontre d'Israël, alors que les opérationssuicides du mois de mars suivant conduisirent nombre de Palestiniens à s'insurger contre les méthodes du Hamas ${ }^{21}$. Le choix de cibles civiles fut singulièrement contesté tandis qu'étaient réprouvées les conséquences des opérations en matière de bouclage et de répression. Le mouvement islamiste fut d'ailleurs, à cette occasion, accusé par les sympathisants du Fatah d'avoir servi les intérêts d'Israël en remettant en cause la cohésion nationale et d'avoir précipité l'arrivée au pouvoir du Likoud ${ }^{22}$. Yasser Arafat, après l'assassinat de Yahia Ayache, avait rendu personnellement visite au Docteur Mahmoud Ez-Zahar (porte-parole du Hamas à Gaza) pour lui présenter ses 
condoléances, et fait tirer une salve d'honneur par la police palestinienne lors des funérailles de " l'ingénieur ". A l'inverse, en mars 1996, il brisera un tabou en traitant les responsables de l'opération de " terroristes ". La branche armée du parti islamiste n'a, jusqu'en mars 1997, revendiqué aucun nouvel attentat.

On peut en déduire que, mises à part les opérations spontanées et incontrôlées, les responsables militaires du Hamas ne sont passés à l'action armée, jusqu'au printemps 1996, que parce qu'ils percevaient un appui de l'opinion publique. Il en est allé de même en mars 1997 lorsque, suite à la décision du gouvernement israélien de construire une colonie juive au Djebel Abou Ghneim (Har Homa) dans la partie arabe de Jérusalem, l'aile radicale du Hamas a bénéficié du soutien tacite d'une opinion publique palestinienne de plus en plus radicalisée par la " réticence " des autorités israéliennes à appliquer les dispositions prévues par les accords de paix. Aussi bien, l'attentat de Tel Aviv du 21 mars 1997 a-t-il été condamné par les responsables de l'Autorité Nationale Palestinienne dans des termes bien différents de ceux utilisés lors de la vague d'attentats du mois de mars de l'année précédente. Considérée comme une réaction inévitable aux " provocations " israéliennes, l'opération du Hamas est alors qualifiée par la presse palestinienne " d'action-suicide " et non plus " d'action terroriste meurtrière "23.

Le maniement de la violence par certains groupes radicalisés, ne suggère pas moins un début de dégradation du mouvement islamiste en un anti-mouvement se situant à michemin entre mouvement social et terrorisme. Ne pouvant envisager ne serait-ce que l'éventualité d'un compromis avec l'Etat d'Israël, les groupes originaires d'Hébron sous occupation israélienne jusqu'en janvier 1997 - présumés responsables des attentats du mois de mars 1996, incarnent en effet, eu égard à leur éloignement progressif de toute base, une forme de plus en plus déstructurée du mouvement social islamiste ${ }^{24}$. Et c'est dans la mesure où ils anticipent sur un compromis Fatah-Hamas qui sonnerait leur glas, que les commandos les plus irréductibles du Djihad Islamique et des brigades Azzeddine El-Qassem prétendent incarner une avant-garde révolutionnaire éclairée et vertueuse, investie de la mission de libérer, par une rupture radicale, la société et ses dirigeants de l'état de jahiliyya dont ils sont prisonniers. Une étape supplémentaire dans l'inversion n'est pas à exclure, qui pourrait voir certains despérados s'en prendre à des dirigeants palestiniens qualifiés " d'impies " et décrits comme de purs et simples successeurs de l'occupant israélien.

L'acteur impossible

L'inaptitude de l'islam politique à doter ses militants d'une identité sociale individuelle et les difficultés qu'éprouvent ces derniers à se construire en tant que sujets personnels, expliquent aussi largement le recours à la violence de certains acteurs islamistes radicaux.

La subjectivation par l'islam, qu'on a décrit comme une des causes de l'adhésion populaire au Hamas du temps de l'Intifada et de la nostalgie qui a persisté de cette époque dans les milieux les plus défavorisés et dans les couches d'âge les plus jeunes, ne s'est en effet pas traduite par la promotion d'individualités, mais par la création et l'entretien d'un climat communautaire. Or, la constitution d'un champ politique moderne implique bien une telle individuation. Et si elle n'exclut pas la mise en jeu de solidarités collectives, elle exclut la perpétuation des habitudes de repli ou d'exaltation identitaire. Ce sont au contraire de telles habitudes qui, par un jeu de miroir, 
nourrissent le culte des martyrs, l'envie de prendre leur place et de s'unir à eux dans le sacrifice.

Le martyrisme : entre expressivité et instrumentalité

Le phénomène des attentats-suicides, étranger aux années d'Intifada, survient au printemps 1994 après le massacre d'Hébron. Son renouvellement se révélera systématiquement lié à la survenue d'événements majeurs attestant de l'impuissance des forces politiques palestiniennes à s'affirmer face à l'occupant ${ }^{25}$.

Le phénomène des attentats-suicides n'est pas seulement symptomatique de la résistance à l'abandon de la lutte armée de la part de certains éléments d'un mouvement para-militaire contraint à la reconversion. Il l'est aussi d'une impossible conversion des acteurs, pris individuellement, à la nouvelle donne créée par les accords d'Oslo.

Le martyrisme palestinien, pour spectaculaires qu'en aient été les manifestations, ne revêt pas l'ampleur repérable dans le cas du Bassidji iranien ${ }^{26}$. Mais il présente, d'une certaine manière, les mêmes caractéristiques de fusion éperdue jusque dans l'anéantissement, et d'identification à une cause collective à laquelle l'effondrement des espérances révolutionnaires et égalitaires engendrées par l'Intifada ne semble plus permettre de manifester son adhésion autrement que par l'immolation. Non seulement la vie ici-bas paraît, aux yeux de nombre de jeunes Palestiniens, dévalorisée par un environnement de " trahison " et de poursuite de la répression, par la disparition de toute opportunité de construire un avenir différent, mais plus aucune échappatoire n'est perçue à la perte de sens, qu'un témoignage extrême ayant à la fois valeur de refus du présent et de solidarité avec le groupe opprimé.

De fait, le militantisme islamiste conduit à la formation d'acteurs non proprement sociaux, certes insérés dans des relations sociales, mais ne se définissant pas en termes de position dans la structure sociale. L'engagement dans la mouvance islamiste obéit à des formes de rationalisation qui permettent de mobiliser une identité culturelle. Mais, étant donné que la réalisation de soi ne s'opère qu'à travers le clan auquel l'acteur appartient, l'islamisme échoue à le doter d'une identité sociale personnelle et à lui donner le sentiment d'exister comme sujet de sa propre trajectoire. Ressource morale et fondement d'une revendication de participation, l'islamisme constitue un obstacle à la naissance d'un véritable sujet. Le contrôle social qu'il instaure, en particulier dans sa dimension de répression sexuelle, ajoute à cet égard au poids des références essentiellement collectives ou communautaires qu'il propose.

Dès lors, les candidats au martyre diluent le paradoxe de l'acteur islamiste dans une violence de type métapolitique où les enjeux politiques semblent subordonnés à des enjeux culturels.

Pour liés qu'ils soient à un événement politique concret, les attentats-suicides n'en relèvent pas moins, chez ceux qui les perpètrent, de la volonté, à défaut de pouvoir se réaliser ici-bas, d'y parvenir dans un au-delà idéalisé. Le jeune candidat au martyre aspire tout à la fois à l'annihilation de Soi et de l'Autre, et à la réunion aux Siens. Assimilé aux souffrances subies, l'adversaire se mue en ennemi et acquiert une identité sub-humaine et idéologisée qui rend son élimination nécessaire, même au prix de la sienne propre.

Au surplus, fascinés par la mort des précédents " martyrs " dont les portraits ornent les murs des localités palestiniennes et dont les noms sonnent familièrement aux oreilles 
de l'ensemble de la population des quartiers défavorisés, les candidats au suicide se persuadent qu'ils marchent dans leurs traces et répondent en quelque sorte à leur appel pour en assurer la relève. Ils sont, dans cette perspective, pris en charge par des groupes armés qui n'ont aucun mal à instrumentaliser cet état d'esprit en cultivant, en particulier, le sentiment de culpabilité qui en constitue une composante. Convaincus d'avoir une responsabilité dans l'échec de l'utopie islamiste des années de l'Intifada, alors même que certains de leurs camarades de combat se sont offerts en sacrifice à la " cause ", les jeunes ainsi enrôlés trouveront tout naturel d'avoir à offrir leur vie pour rester dignes de leurs aînés disparus. L'organisation revendiquant les opérationssuicide y gagne en prestige auprès de la base la plus radicalisée de la mouvance, la capacité d'une organisation à aligner des martyrs augmentant son capital social et symbolique dans les quartiers populaires.

" Le monde est un paradis pour l'infidèle mais une prison pour le croyant "27

Hisham Mohammed correspond au portrait-type du sujet impossible, frustré des promesses de la libération et de la modernité, happé par l'exemplarité d'un camarade qui l'a précédé dans le sacrifice.

Par un méchant jour de novembre 1994, Hisham Mohammed, qui jeûne depuis le matin en prévision de son immolation, enfourche sa bicyclette et se rend au check point israélien situé à l'entrée de la colonie juive de Netzarim dans la bande de Gaza. Arrivé à hauteur des soldats, il fait exploser la bombe artisanale qu'il a consciencieusement arrimée à son engin, entraînant trois soldats israéliens dans sa mort. Hisham Mohammed avait vingt ans ${ }^{28}$.

Sa dépouille est aussitôt escamotée par des membres des services de sécurité palestiniens qui inhument le jeune homme dans la plus grande discrétion. L'heure est aux négociations de paix avec Israël. Point n'est besoin, aux yeux de l'Autorité Palestinienne, d'entretenir davantage le culte des " martyrs ". Dans le quartier populaire de cheikh Redwane, dont Hisham était originaire, on ne l'entend pas de cette oreille. Le vendredi suivant, soit le 18 novembre 1994, les militants islamistes réunis autour de la mosquée Palestine boivent les paroles du cheikh Baher, l'un des prédicateurs les plus éloquents du Hamas. Leurs dévotions achevées, iIs décident de se rendre au domicile de Hisham pour y présenter leurs condoléances à sa famille. Las! Les forces anti-émeutes encerclent la mosquée et entravent la procession. Des pierres fusent. Les policiers palestiniens ouvrent le feu. Les affrontements entre forces de l'ordre et manifestants s'étendent aux artères principales de la ville de Gaza jusqu'à gagner le quartier de cheikh Redwane. On relève, à l'issue de ce fameux " vendredi noir ", les corps de treize manifestants, tandis que quelque deux cents blessés affluent vers les hôpitaux de la ville. Pour la première fois de sa brève existence, l'Autorité Palestinienne décrète un couvre-feu.

Quelques jours avant sa mort, Hisham avait pris soin de rendre visite à sa soeur.

" Il est allé voir sa soeur et lui a offert un cadeau symbolique. Sa soeur l'a averti : " Si tu m'apportes un cadeau à chaque fois que tu me rends visite, je ne voudrais plus que tu viennes ". Il lui a alors répondu " considères que je viens pour la dernière fois ". Mais elle n'a pas réalisé ce que cela signifiait "29.

Pas plus que sa soeur, les autres membres de la famille de Hisham n'avaient perçu sa détermination. Elle avait pourtant été entière, de venger l'assassinat par le Shin Beth, le 2 novembre précédent, de Hani Abed, l'un des membres actifs du Djihad Islamique à Gaza, faisant suite à celui de son meilleur ami au mois d'avril précédent. La 
photographie de ce camarade porte encore l'inscription que Hisham avait tracée de sa main quelques jours avant sa mort : " Je jure devant Dieu de venger ta mort mon frère ".

" Malgré la présence de l'Autorité Palestinienne, il voyait tous les jours les juifs à Gaza. Il disait que c'était la paix, mais que les Israéliens étaient toujours là. C'est pourquoi il a choisi d'attaquer une colonie. Son meilleur ami venait d'être tué par le Shin Beth à Khan Younis alors que l'Autorité Palestinienne était déjà là. Beaucoup de ses amis avaient le même caractère. Deux de ses amis, qui nous ont aidé à construire sa tombe, se sont tués après lui. Ils sont aussi shahid (martyrs). Il y en avait beaucoup comme lui. Il voulait venger ses amis avant tout. Il voulait tenir la promesse de la vengeance. Et il savait aussi que combattre les Israéliens est un devoir national. Il savait que les Israéliens ne nous donneraient pas nos droits. Comme maintenant: les Israéliens ne nous donnent rien et installent des colonies partout. Notre frère aîné avait aussi été arrêté. Hisham avait une haine personnelle contre les sionistes. Son frère avait été arrêté avec d'autres et avait été battu. Il conservait des cicatrices. L'un de ceux qui avait été arrêté avec lui était mort sous la torture. C'est aussi une des raisons pour lesquelles Hisham les haïssait ".

Issu d'une famille de réfugiés, Hisham, après avoir, à l'instar de nombre de jeunes de son âge, suspendu sa scolarité alors qu'il était encore dans le primaire, rejoint l'entreprise de plâtrerie familiale. Sa famille, installée auparavant dans le camp de Chati, a été relogée dans le " housing project " du quartier de cheikh Redwane. Militant du Hamas durant l'Intifada à laquelle il avait participé activement, et durant laquelle il avait bravé régulièrement le couvre-feu imposé par l'armée d'occupation, il avait été arrêté pour avoir jeté des pierres sur des soldats, et emprisonné durant six mois. C'est durant ce " séjour " dans les geôles israéliennes qu'il avait adhéré au Djihad Islamique pour " participer plus rapidement à une opération ".

Devenu imam de la mosquée de son quartier, une modeste bâtisse construite de ciment et de tôle ondulée comme on en trouve couramment dans les camps de réfugiés, et qui porte aujourd'hui son nom, Hisham s'était consacré à ses oeuvres et à sa préparation au " grand jour " tant sa situation de domination lui était devenue humiliante et intolérable. A sa mère, qui caressait le projet de lui construire une maison en prévision d'un futur mariage, il répondit qu'il se marierait certes, mais " à une hourie " (du nom des vierges, promises aux croyants, qui peuplent le paradis).

Désemparé par l'interruption de la lutte armée et sceptique quant à ses chances de " trouver son compte " dans le processus de paix, Hisham ne portait pas la même appréciation que ses frères à l'égard de l'Autorité Palestinienne : " Mon frère est arrivé à ce type de conclusion après avoir lu et compris profondément le Coran. Moi, je suis une personne simple. Je prie, mais je n'ai pas la même compréhension du Coran. Je n'étais pas tout à fait d'accord avec lui. Je lui disais qu'il fallait donner une chance à l'Autorité Palestinienne de faire quelque chose de bien pour les Palestiniens. Mais, pour lui, l'action militaire ne devait jamais cesser. Il était totalement convaincu que les Israéliens se moquaient des Palestiniens. Il décrivait l'Autorité comme une illusion ".

Plus taciturne que jamais après l'assassinat de son meilleur ami, et ne pouvant se résoudre à la défaite de la " génération de l'Intifada ", Hisham agit comme s'il avait fait partie de quelque société secrète. Transmises par le Djihad Islamique à sa famille après sa mort, des photographies de cette époque découvrent une facette insoupçonnée de ses activités. Présentant, quelques mois auparavant, les traits d'un adolescent au visage 
glabre, Hisham arbore désormais l'allure d'un " soldat " trop vite monté en graine. Tenant en joue une cible imaginaire, le jeune homme prend la pose la kalachnikov à la hanche.

" Quand son meilleur ami a été assassiné, il a commencé à s'absenter de son boulot. Je lui ai dit: " Ecoute, je peux comprendre que tu t'absentes deux ou trois jours car ton ami est mort. Mais, dorénavant, si tu ne retournes pas travailler, j'arrêterai de te donner de l'argent. Tu vas perdre cinquante chekels par jour ". Il m'a répondu: " Je m'en fiche, je ne reviendrai pas ". Pour lui, l'argent et la vie n'avaient pas d'importance ".

Hisham s'applique alors à entretenir quasi mystiquement le culte de son ami, et à inscrire des sentences annonciatrices de sa vengeance, sur les murs faisant face à la demeure de ses parents. Il prend également soin, pour expliquer son geste, d'écrire une lettre à sa famille et d'enregistrer une cassette vidéo appelant les musulmans à lutter contre Israël jusqu'au recouvrement de la terre de Palestine. La vengeance est devenue une exigence morale lui incombant personnellement. Il se sent investi de la mission de laver les humiliations infligées quotidiennement à ses compatriotes et d'élever ces derniers à une nouvelle dignité.

Conclusion

C'est un trait général des mouvements islamistes que d'être pris dans une tension entre la nécessité d'entretenir la ferveur dont ils deviennent progressivement le seul support, du fait des désaveux infligés aux organisations nationalistes " laïques ", et celle de ne pas se couper des jeux politiques au sein desquels ils aspirent à s'intégrer. La violence leur apparaît, dans ces conditions, à la fois comme une menace et comme une nécessité. Les mouvements islamistes ayant vocation à convertir leur capital social en ressource politique ne peuvent en effet ni la cautionner exagérément, au risque de se priver de la possibilité d'exister durablement dans le système institutionnel, ni la condamner, sous peine de se couper d'une partie de leur base et de perdre leur capacité de contribuer à la destabilisation des régimes ou des forces en place. Aussi, de plus en plus dépourvue des significations politiques et sociales qu'avaient revêtues les actions armées du Hamas du temps de l'Intifada, la violence des commandos-suicides échappe-t-elle désormais en partie au contrôle du parti islamiste. Et, si celui-ci continue de chanter les louanges de ses " martyrs ", il ne qualifie plus désormais leur " sacrifice " comme ayant une fin coïncidant avec sa propre vocation politique et sociale.

Cette dissociation entre la logique institutionnelle du parti islamiste et la logique armée d'une fraction de la base radicalisée du mouvement explique l'actuelle absence de cohérence des initiatives du Hamas. La récente dégradation des relations israélopalestiniennes pourrait cependant, en réactualisant les mots d'ordre de la résistance armée, ressouder les rangs de l'organisation islamiste.

Sans doute les affrontements armés de septembre 1996 ne condamnent-ils pas définitivement les accords d'Oslo à l'échec. Ils ont néanmoins fortement entamé les espoirs qu'avait placé la majorité de la société palestinienne dans le processus de paix.

Prise en tenailles entre l'intransigeance israélienne et les exigences de ses bailleurs de fonds, l'Autorité Palestinienne se retrouve prisonnière d'un processus de paix conçus par ses initiateurs palestiniens en vue d'arracher la création d'un Etat souverain, si réduit soit-il, mais, même ce résultat demeure incertain. 
Horizon indépassable pour un Yasser Arafat soucieux de préserver son leadership et hanté par l'idée qu'une explosion incontrôlable de violence dans ses territoires puisse être écrasée par Israël et le contraindre à un nouvel exil, les accords d'Oslo incarneront-ils encore longtemps le " rêve palestinien " aux yeux d'une population dont les conditions d'existence ne cessent de se détériorer et dont la colère et le désespoir ne cessent de croître ? Les islamistes du Hamas en doutent, qui attendent le " naufrage " du processus de paix et l'éventuel échec de l'Autorité palestinienne pour fourbir à nouveau leurs armes. Bénéficiaire potentiel des ratés du processus de paix, le Hamas risque en effet de capitaliser également le ressentiment qu'une partie de la population tend à nourrir à l'égard d'une Autorité Nationale Palestinienne tenue pour responsable de son aliénation politique et économique.

L'islamisme palestinien représente, on le voit, une force avec laquelle tout scénario politique sera, à l'avenir, contraint de composer. Yasser Arafat ne saurait promettre à Israël la garantie de sa sécurité en dehors d'une concertation avec des islamistes dont la capacité de nuisance n'a pas été définitivement entamée. L'Etat d'Israël l'a d'ailleurs compris, qui a, en plusieurs occasions, noué des contacts officieux avec le Hamas.

Prisonnier de son " protectorat " de Gaza, soucieux de ne pas provoquer une guerre civile palestinienne susceptible de servir de prétexte à une intervention de l'armée israélienne dans les territoires autonomes, Yasser Arafat pourrait lui aussi être amené à rechercher un compromis avec la mouvance islamiste en cas de confrontation avec un gouvernement Israëlien ${ }^{30}$ qui semble faire actuellement le pari d'entraîner l'Autorité Palestinienne dans une stratégie de la tension susceptible de déboucher sur une nouvelle logique de violence. Toute la question serait alors du degré d'adhésion au recours à la violence que Yasser Arafat serait lui-même obligé de confesser. Entre une acceptation impossible et une condamnation ouvrant la voie à de nouvelles ruptures, les voies possibles d'une instrumentalisation de la violence islamiste par l'Autorité Palestinienne sont étroites.

* Séverine Labat est chargée de recherches au CADIS

\section{NOTES}

1. Ziad Abu Amr, Islamic fundamentalism in the West Bank and Gaza : Muslim Brotherhood and Islamic Djihad, op. cit.

2. " Faucons " du Fatah, " Aigles rouges " du FPLP, cellules " Seif Al-Islam " du Djihad Islamique.

3. Entretien avec Ghazi Hamad à Gaza le 23 septembre 1996.

4. François Burgat, L'islamisme en face, op. cit., l'idéologie islamiste considère le djihad contre l'ennemi extérieur comme allant de pair avec la réislamisation (au besoin " musclée ") de la société palestinienne, d'où la coexistence entre les deux formes de violences. A cet égard, voir également Ziad Abu Amr, Islamic fundamentalisme in the West Bank and Gaza : Muslim Brotherhood and Islamic Djihad, op. cit. 
5. Voir Edward Saïd, Peace and Its Discontents. Gaza-Jericho 1993-1995, London, Vintage, $197 \mathrm{p}$.

6. Sur les fondements et les conditions de l'accord de paix, voir Ghassan Salamé (dir.), Proche-Orient : les exigences de la paix, Complexe, Bruxelles, 1994, $167 \mathrm{p}$.

7. . Entretien à Gaza le 23 septembre, avec Rachid, militant du Hamas proche des commandos Azzeddine El-Qassem. Agé d'une trentaine d'années, Rachid a abandonné ses études avant le bac. Il travaille comme employé dans un magasin. Accusé d'assurer la coordonation entre différentes cellules du Hamas, il fut arrêté et torturé deux fois, en 1981 et 1983.

8. A ce sujet, voir Elias Sanbar, " L'autogouvernement palestinien " in Ghassan Salamé (dir.), Proche-Orient : les exigences de la paix, op. cit, p. 101-110.

9. Entretien à Gaza le 24 septembre, avec Nasser militant du Hamas durant l'Intifada. Agé d'une trentaine d'années, Nasser est issu d'une famille de bédouins originaires du Sinaï, installée dans la région de Beer Sheva, puis réfugiée à Gaza. Ne disposant pas du statut de réfugié dûment enregistré auprès de l'UNRWA, Nasser a toutefois pu suivre l'enseignement d'une école de l'organisme international chargé des réfugiés. Inscrit, durant l'Intifada, à l'université islamique de Gaza, il suit de loin des cours de char'ia, consacrant l'esentiel de son temps à l'activisme anti-israélien (inscription de graffitis, jets de pierres).

10. En raison, notamment, du poids des réfugiés dans la population, de l'influence des Frères Musulmans égyptiens, mais également de l'enclavement et de l'isolement dont souffre la bande de Gaza, d'où la facilité avec laquelle les islamistes sont parvenus à imposer un sévère contrôle social. On en veut pour preuve la récente interdiction, par le maire Fatah de Gaza, de la vente de boissons alccolisées dans les restaurants et bars de la ville.

11. Entretien avec Ghazi Hamad à Gaza le 23 septembre 1996.

12. Déconvenues semblant tenir autant à la mauvaise volonté des autorités israéliennes dans la mise en oeuvre des accords que dans les faiblesses et les ambiguités mêmes d'un accord signé sous contrainte. A ce sujet, voir le Rapport de la Fédération Internationale des Ligues des Droits de l'Homme (FIDH) : " En désespoir de paix ", mars 1997. Voir également Edward W. Saïd, " Victimes consentantes ", Le Monde Diplomatique, novembre 1994.

13. Le premier ministre Shimon Pérès en reporte sine die l'application le 28 mars 1996, suite à la vague d'attentats-suicides perpétrés en Israël par des militants du Hamas.

14. La " lettre d'accompagnement " (ou "lettre de garantie") américaine à cet accord affirme en effet que " l'étendue de chacun des redéploiements (ces derniers devant intervenir en mars et septembre 1997 puis en août 1998) sera fixée par Israël en fonction de ses besoins de sécurité ". A cet égard, voir Le Monde du 16 janvier 1997. 15. Bouclage ayant des répercussions économiques catastrophiques à Gaza où s'entassent plusd'un million d'habitants dont $70 \%$ ont moins de 20 ans et dont $75 \%$ sont des réfugiés : chute de $30 \%$ du revenu par habitant entre février 1996 et septembre 1996 ; taux de chômage de 50 à près de 80 \% selon les époques.

16. Voir Geoffrey Aronson, " Pendant la négociation, la colonisation continue ", Le Monde Diplomatique, Novembre 1996, p 4-5. Hors Jérusalem-Est, plus de 160000 colons juifs se répartissent entre Gaza (qui en compte 8000 ) et la Cisjordanie (environ 150 000), soit une hausse de $49 \%$ entre juin 1992 et mai 1996, c'est-à-dire sous le gouvernement travailliste. 
17. Pierre Haski, " L'espoir bouclé des Palestiniens de Gaza : la zone autonome est devenue une vaste prison à ciel ouvert " in Libération, 6 octobre 1996.

18. Jérusalem-Est, avec environ 200000 colons (soit une hausse de $33 \%$ entre juin 92 et mai 96), est désormais à majorité juive.

19. A cet égard, voir Jean-François Legrain, "Judaïsation et démembrement : politiques israéliennes du territoire en Cisjordanie-Gaza (1967-1995) " in Monde Arabe MaghrebMachrek, n 152, avril-juin 1996, p. 42-73.

20. Les opérations militaires du Hamas ne concernaient que les territoires occupés avant la signature des accords d'Oslo. Depuis lors, elles se sont étendues au territoire d'Israël.

21. Les manifestations contre le " terrorisme " (largement organisées par le Fatah) se sont cependant vite transformées en manifestations contre le bouclage qui sévit depuis le mois de mars 1996.

22. Ce qui constitue une perte de popularité à court terme, mais peut consitituer un gain à moyen terme dans la mesure où l'intransigeance du Likoud peut remobilser la base du Hamas contre Israël.

23. A ce sujet, voir l'article de Dany Rubinstein : " Territoires occupés : maintenant la rue soutient à nouveau le Hamas ", paru dans le journal israélien Ha'aretz et reproduit dans Courrier International, n 334, du 27 mars au 2 avril 1997.

24. Pour une définition de l'antimouvement social, voir Michel Wieviorka, Sociétés et terrorisme, Paris, Fayard, 1988.

25. Le massacre d'Hébron perpétré par Baruch Goldstein le 25 février 1994, est, de fait, l'élément déclencheur de la vague d'attentats du printemps 1994 ; celle de l'automne 1994 fait suite à l'affaire Wachsman et à l'assassinat, par le Shin Beth, d'un des chefs du Djihad Islamique à Gaza ; celles du printemps et de l'été 1995 répondent à l'attentat à l'explosif perpétré par les services israéliens contre l'un des chefs des Brigades Azzeddine El-Qassem à Gaza ; celles de novembre 1995, puis de février et mars 1996 sont provoquées par les assassinats consécutifs de Fathi Chiqaqi (chef du Djihad Islamique assassiné par le Mossad à Malte en octobre) et de Yahia Ayache, tandis que l'attentat de mars 1997, à Tel Aviv, fait suite à la décision du gouvernement israélien de construire une implantation juive au Djebel Abou Ghneim (Har Homa) dans la partie arabe de Jérusalem.

26. A cet égard, voir dans ce numéro de Cultures \& Conflits, la contribution de Farhad Khosrokhavar, " Le modèle Bassidji ".

27. Cité par Beverley Milton-Edwards, Islamic Politics in Palestine, Tauris Academic Studies, Londres, 1996, p. 167.

28. On trouvera un autre portrait de Hisham signé de Patrice Claude dans Le Monde du 11 avril 1994.

29. Entretien avec les frères de Hisham Mohammed le 25 septembre 1996 à Gaza. 30. En témoigne la rencontre, à Naplouse, fin février 1997, entre Yasser Arafat et l'ensemble des formations politiques palestiniennes (Hamas compris) en vue de la promotion d'un " dialogue national ". 
INDEX

Thèmes : Fatah (Palestine), Hamas (Palestine), OLP

Index chronologique : 1990 - 2000

Mots-clés : guérillas et organisations clandestines, kamikaze (missions suicide), religion, violence

Index géographique : Israël, Palestine 